\title{
L'IRSN et la surveillance de la radioactivité de l'environnement en France : état des lieux et perspectives
}

\author{
D. CHAMPION ${ }^{1}$, J.-M. PERES ${ }^{1}$
}

(Manuscrit reçu le 6 février 2009, accepté le 26 mars 2009)

RÉSUMÉ Le dispositif de surveillance de la radioactivité de l'environnement, exploité aujourd'hui par l'IRSN, est le fruit d'un développement historique et graduel, démarré à la fin des années 50. Au départ, la mise en place d'une surveillance radiologique du territoire visait à mesurer les retombées radioactives des essais nucléaires atmosphériques réalisés dans l'hémisphère nord. Cette surveillance s'est renforcée à partir des années 70, avec le développement du parc électronucléaire français, pour aboutir à un ensemble de réseaux permanents qui fournissent de nombreux résultats de mesure, en temps réel dans le cas de la télésurveillance, ou dans des délais aussi courts que possible dans le cas des analyses d'échantillons en laboratoires. L'IRSN estime aujourd'hui que les dispositifs de surveillance et leur déploiement doivent s'adapter aux évolutions des techniques, des connaissances de l'état radiologique de l'environnement et des besoins nouveaux exprimés par la société.

ABSTRACT IRSN and monitoring of radioactivity in the environment in France: state and prospects.

The monitoring of radioactivity in the environment, now operated by IRSN, is the result of historical development and gradual, starting in the late 50s. Initially, the establishment of radiological surveillance of the territory aimed to measure the fallout of atmospheric nuclear tests conducted in the northern hemisphere. This monitoring has increased from 70s, with the development of French nuclear power park, leading to permanent networks that provide many measurement results in real time in the case of On-line networks, or in time as short as possible in the case of analysis of samples in laboratories. IRSN believes today that the monitoring devices and their deployment must adapt to changing technology, knowledge of the radiological condition of the environment and the needs expressed by society.

Keywords: monitoring / radioactivity / environment

\section{Introduction}

Le décret du 22 février 2002 définissant les missions de l'Institut de radioprotection et de sûreté nucléaire (IRSN) indique, dans son article 1-II, que l'institut «participe à la veille permanente en matière de radioprotection,

\footnotetext{
1 IRSN, Direction de l'Environnement et de l'Intervention, 35-31 rue de l'écluse, 78116 Le Vésinet Cedex, France.
} 
notamment en concourant à la surveillance radiologique de l'environnement... ». Cette activité de surveillance n'est pas née avec le nouvel institut mais est profondément ancrée dans l'histoire des entités qui en sont à l'origine : principalement le SCPRI ${ }^{2}$ devenu OPRI ${ }^{3}$, mais aussi l'IPSN ${ }^{4}$ au travers de ses activités d'étude en radioécologie et de suivi de la radioactivité en Polynésie française. De ce fait, l'état du dispositif de surveillance et des moyens techniques et humains actuellement mobilisés par l'IRSN est principalement le reflet de cette histoire.

Fort de cet héritage solide, l'IRSN estime aujourd'hui que cette activité doit bénéficier d'une réflexion actualisée, tenant compte de l'évolution des techniques, des connaissances de l'état radiologique de l'environnement, des questions nouvelles posées à l'IRSN et des attentes des différents acteurs de la société. Ainsi, le Contrat d'objectifs signé en 2006 entre l'IRSN et ses cinq ministres de tutelle prévoit explicitement l'engagement d'un plan de modernisation et d'optimisation de ses réseaux de surveillance, basé sur une stratégie actualisée et incluant le renforcement de la valorisation des résultats de la surveillance.

\section{L'ancrage historique de la surveillance de la radioactivité dans l'environnement}

La surveillance de la radioactivité de l'environnement réalisée par l'IRSN repose sur la mise en place et l'exploitation d'un ensemble de stations de prélèvements et de mesures déployées sur le territoire national, y compris l'outre-mer. Grâce à cet ensemble, l'Institut dispose ainsi d'une connaissance de l'état radiologique de l'environnement permettant notamment de répondre à trois objectifs complémentaires :

- contribuer à vérifier que les activités nucléaires sont menées dans le respect des règles de rejet qui s'imposent à elles ;

- s'assurer, plus généralement, que le territoire, dans toutes ses composantes, reste dans un état radiologique satisfaisant qui n'induit pas une exposition excessive des personnes ou des écosystèmes ;

- détecter aussi rapidement que possible et caractériser une élévation anormale de radioactivité qui pourrait résulter d'un événement radiologique ou nucléaire survenant en France ou à l'étranger.

\footnotetext{
2 Service central de protection contre les rayonnements ionisants, responsable du contrôle de la radioprotection en France jusqu'en 1994.

3 Office de protection contre les rayonnements ionisants, ayant succédé au SCPRI à partir de 1994 jusqu'à la création de l'IRSN en 2002.

4 Institut de protection et de sûreté nucléaire, rattaché au CEA jusqu'à la création de l'IRSN en 2002.
} 
Ces objectifs déterminent deux fonctions complémentaires attendues de la part de ce dispositif de surveillance : une fonction de vigilance, visant à déceler aussi rapidement que possible une élévation inhabituelle de la radioactivité dans l'environnement, le cas échéant avec un rôle d'alerte; une fonction de caractérisation des expositions de la population liées à l'environnement, en situation normale et en cas d'accident. Comme on le verra plus loin, ces deux fonctions n'appellent pas nécessairement les mêmes besoins techniques.

\subsection{L'héritage du SCPRI et de l'OPRI}

L'essentiel du dispositif de surveillance exploité aujourd'hui par l'IRSN est le fruit d'une construction historique graduelle, réalisée par le SCPRI puis l'OPRI, dès les années 50. À cette époque, et jusqu'à la fin des années 70, la France, comme les autres pays européens, était régulièrement impactée par les retombées radioactives des essais nucléaires atmosphériques réalisés dans l'hémisphère nord, principalement par les États-Unis et l'Union Soviétique, puis la Chine. Ce contexte particulier a déterminé une bonne part du développement des réseaux de surveillance du SCPRI, notamment par le prélèvement d'échantillons des constituants atmosphériques (aérosols, eau de pluie) et de la chaîne alimentaire. Le développement du dispositif de surveillance a également accompagné, à partir des années 60, celui des centres nucléaires de recherche, puis celui du parc électronucléaire français, déterminant une concentration des points de prélèvements et de mesures autour des principaux sites nucléaires. Enfin, l'accident de Tchernobyl, survenu en 1986, a conduit à renforcer le rôle d' alerte de la surveillance radiologique, principalement au travers du développement du réseau TELERAY mis en place dans les années 90 pour mesurer en temps réel le débit de dose gamma ambiant. Au cours de cette période, le SCPRI, puis l'OPRI, ont ainsi acquis de précieuses chroniques de mesures des principaux radionucléides alors présents dans l'environnement français.

Comme dans tous les pays nucléarisés, cette surveillance est fondée sur un principe de mesures de « routine » adossées à un ensemble de stations de prélèvement d'échantillons et de stations de télémesure qui permettent de disposer de résultats à une fréquence régulière, voire en continu pour certains paramètres. Néanmoins, le dimensionnement de ces réseaux et les caractéristiques des dispositifs les constituant sont variables d'un pays à l'autre (De Cort, 2007). Privilégiant la rapidité d'acquisition, les performances métrologiques des appareils permettent de vérifier l'absence de risque sanitaire, de détecter des évolutions anormales significatives du niveau de radioactivité dans l'environnement ou de donner l'alerte.

Ainsi, le dispositif de surveillance radiologique du territoire mis en œuvre aujourd'hui par l'IRSN comprend :

- des réseaux de prélèvement d'échantillons de l'environnement ;

- des réseaux de télésurveillances automatisés. 
Les réseaux de prélèvement d'échantillons de l'environnement. Les échantillons prélevés sont ensuite analysés dans les laboratoires de l'IRSN au Vésinet : environ 600 stations couvrent le territoire et fournissent chaque année plus de 30000 prélèvements de 20 types différents, sur lesquels sont pratiquées 54000 analyses de radioactivité. La grande majorité de ces échantillons (78 \%) est constituée de prélèvements d'aérosols assurés par le réseau dit « $\mathrm{AS}$ » (pour prélèvements d'«Air au Sol») composé de 70 stations (Fig. 1), dont 34 à proximité des installations nucléaires, 25 en sites urbains, 7 sur des sites dits « de référence » et 4 en outre-mer.

Les autres types de prélèvements sont constitués de matrices variées, dans les proportions indiquées dans la figure 2 .

L'ensemble des échantillons provenant des réseaux de surveillance de l'IRSN sont analysés dans les laboratoires de l'IRSN, qui disposent d'accréditations par le COFRAC, garantissant ainsi la qualité métrologique des résultats obtenus. En complément de cette surveillance par prélèvement d'échantillons, l'IRSN mesure le débit de dose gamma ambiant à l'aide de dosimètres thermo-luminescents répartis sur environ 1000 points du territoire et relevés tous les 6 mois.

Les réseaux de télésurveillances automatisés. Ces réseaux, dédiés à la surveillance continue des milieux atmosphérique et aquatique, comprennent :

- TELERAY : réseau dédié à la surveillance en continu du rayonnement gamma ambiant de l'air (19 millions de résultats de mesure par an), avec une fonction d'alerte (relayée vers un agent d'astreinte via un superviseur basé au Vésinet) en cas d'élévation inhabituelle du débit de dose ambiant. Il s'agit du principal réseau de télésurveillance, constitué de 164 balises (tubes Geiger-Muller) réparties ainsi : 38 autour des sites nucléaires, 120 dispersés sur le territoire métropolitain (dont plus d'une douzaine en région parisienne) et 6 dans les DOM-TOM (cf. Fig. 3) ;

- $S A R A$ : réseau dédié à la surveillance en continu de la radioactivité des aérosols atmosphériques, au moyen de balises automatisées de prélèvement et de mesure des aérosols et télétransmission périodique des résultats des analyses vers le superviseur de l'IRSN au Vésinet. En cas d'accident de grande ampleur, ce réseau permettrait de caractériser, pratiquement en temps réel, le spectre radiologique des aérosols prélevés. Cette information serait essentielle pour conforter l'estimation rapide des doses reçues par les personnes exposées au panache radioactif, et pour suivre l'évolution spatiale de ce panache. Ce réseau comporte actuellement 13 balises, dont 11 sur des sites de Météo-France et 2 sur des sites IRSN ;

- HYDROTELERAY : réseau dédié à la surveillance en continu des principaux fleuves, en aval de toutes les installations nucléaires ; il permet de vérifier en 

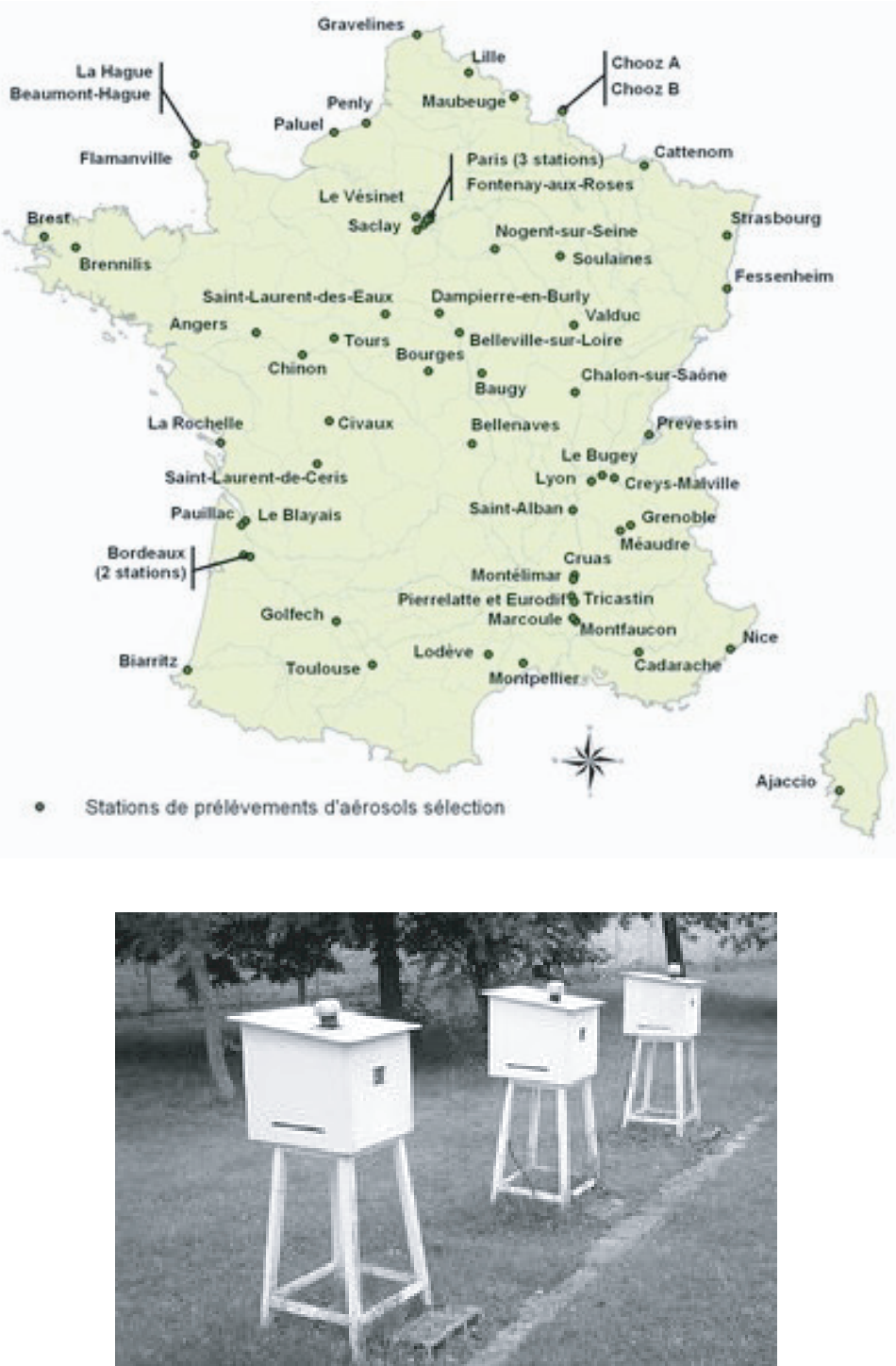

Figure 1 - Carte d'implantation des stations de prélèvement d'aérosols en 2007 et illustration d'une station de prélèvement.

Map of location of aerosol sampling stations in 2007 and illustration of a sampling station. 
Sols, sé diments, boues: $7,4 \%$

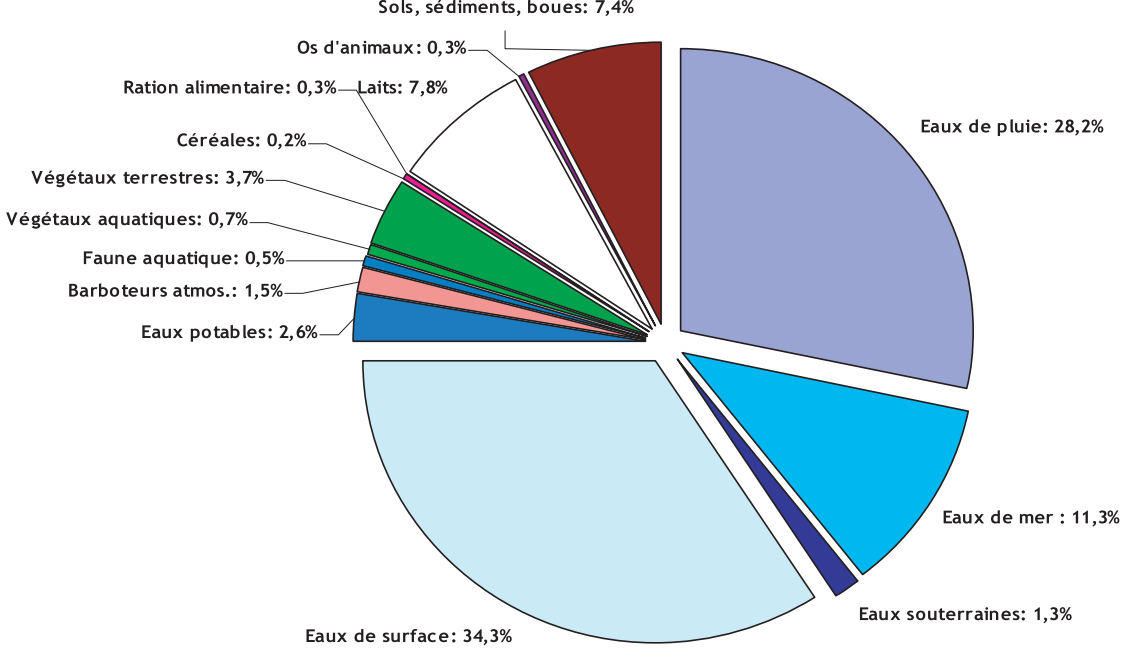

Figure 2 - Proportions de matrices prélevées (hors aérosols) en 2007 dans le cadre de la surveillance de la radioactivité assurée par l'IRSN.

Proportions of sampled matrices (excluding aerosols) in 2007 under the monitoring of radioactivity provided by IRSN.

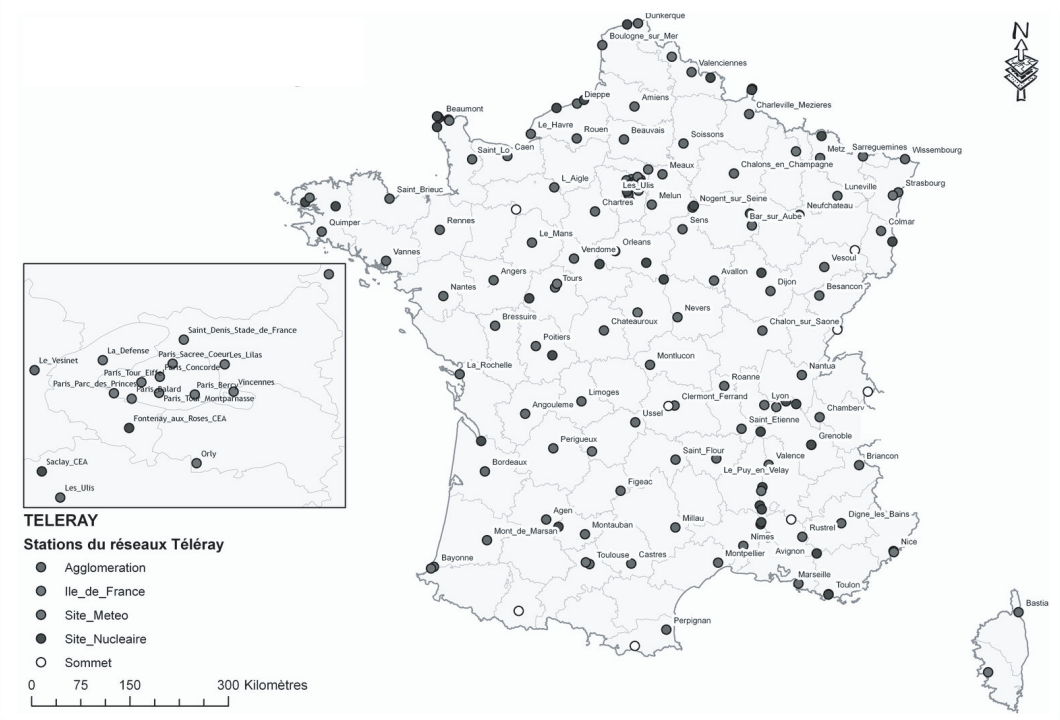

Figure 3 - Répartition territoriale des balises du réseau TELERAY.

Distribution of territorial network TELERAY stations. 
permanence que les eaux de ces fleuves ne présentent pas d'activité anormalement élevée, en lien avec un éventuel rejet accidentel, avant leur sortie du territoire national. Chacune des 7 stations comprend un dispositif de prélèvement continu de l'eau du fleuve, avec un débit de 6 à $10 \mathrm{~m}^{3} / \mathrm{h}$, et une mesure directe par spectrométrie gamma $(\mathrm{NaI})$ avec un temps d'intégration de 2 heures. En cas d'alarme, des prélèvements instantanés sont automatiquement réalisés pendant la durée de l'événement radiologique, pour une analyse fine ultérieure ;

- TELEHYDRO : réseau dédié à la surveillance en continu des radionucléides émetteurs gamma dans les eaux usées au sein des stations d'épuration de grandes agglomérations françaises, à l'aide d'une sonde NaI mesurant l'activité des radionucléides émetteurs gamma. Dans son dimensionnement actuel (7 stations), ce réseau permet de réaliser des observations ponctuelles, notamment en rapport avec les rejets radioactifs des services de médecine nucléaire de centres hospitaliers.

Il convient de souligner certains aspects importants de ce dispositif de surveillance :

- il est marqué par la part largement prépondérante de la surveillance du milieu atmosphérique, assurée par le réseau de prélèvement d'aérosols « AS » et par la télésurveillance (TELERAY et SARA). Cette situation est à la fois le fruit de l'histoire (essais nucléaires atmosphériques et accident de Tchernobyl) et une nécessité propre aux enjeux spécifiques des situations accidentelles, aussi exceptionnelles soient-elles ;

- l'IRSN effectue de nombreuses mesures d'activité globale bêta et alpha, sur les prélèvements d'aérosols (respectivement 32000 et 7000 mesures par an) et les prélèvements d'eau (respectivement 3500 et 1000 mesures par an). Ces mesures présentent l'avantage de pouvoir être réalisées rapidement ; n'étant pas sélectives, elles permettent de détecter une large gamme d'anomalies radiologiques. Elles sont complétées par la caractérisation de radionucléides (plus de 1600 mesures spécifiques du tritium, plus de 150 mesures de carbone-14, plus de 4000 spectrométries gamma par an), avec une limite de détection qui suffit au respect des trois objectifs indiqués au début de ce chapitre. À ce titre, la réalisation de telles mesures en grand nombre répond bien à la fonction de vigilance réactive attendue de la part du dispositif de surveillance mis en œuvre par l'IRSN. En revanche, souvent, ces mesures ne permettent pas de quantifier finement les radionucléides de l'environnement dus aux activités nucléaires et sont d'un intérêt limité pour les études et expertises visant à évaluer les doses reçues par la population ; elles doivent être complétées par des mesures spécifiques de caractérisation; 
- il en va de même pour le réseau TELERAY, qui répond essentiellement à un besoin de dépistage précoce d'une anomalie radiologique mais qui ne permet pas, à lui seul, de déterminer les caractéristiques des sources d'exposition et nécessite l'engagement systématique d'investigations complémentaires en cas de détection d'une anomalie ;

- enfin, en raison de l'étendue territoriale des réseaux de prélèvements de l'IRSN, le fonctionnement opérationnel de ces réseaux dépend d'opérateurs locaux variés, chargés de faire la collecte régulière d'échantillons envoyés ensuite dans les laboratoires de l'IRSN (services techniques des exploitants nucléaires, Météo-France, Marine nationale, services administratifs locaux et services déconcentrés de l'État, Office national interprofessionnel des céréales, exploitants agricoles, particuliers, etc.). Cette dépendance et la multiplicité des acteurs sont une source de fragilité pour une bonne maitrise des opérations de prélèvement par l'IRSN. À ce titre, un effort important est dédié à la formation des correspondants et à la mise en place de modalités de contrôle de la qualité de ces opérations. Cet effort vient compléter une plus grande internalisation de ces opérations.

\subsection{L'héritage de l'IPSN}

L'héritage de l'IPSN relatif à la mission de surveillance de la radioactivité de l'environnement n'est pas quantitativement important au sein du dispositif actuel ; en revanche, il est qualitativement essentiel, et tout-à-fait complémentaire de celui de l'OPRI. D'une manière générale, les activités historiques de l'IPSN concernant la radioactivité de l'environnement sont marquées par des études et des recherches visant à connaître la distribution spatiale et temporelle des substances radioactives dans l'environnement et à évaluer l'exposition des populations qui en résulte. Parmi les actions les plus significatives, il convient de mentionner :

- l'exploitation d'un observatoire permanent de la radioactivité de l'environnement (OPERA), concernant les milieux atmosphérique, terrestre, aquatique et marin, et constitué d'un ensemble de stations de prélèvement, dont certaines sont dotées de dispositifs technologiques sophistiqués (milieux atmosphérique et fluvial). À titre d'illustration, l'observation de la radioactivité de l'air existe aujourd'hui depuis 50 ans et, comme pour le SCPRI, a été principalement motivée par le besoin de détecter et caractériser les arrivées de poussières radioactives résultant des essais nucléaires atmosphériques. $\mathrm{Au}$ cours de cette période d'observation, l'IPSN puis l'IRSN se sont efforcés d'adapter les dispositifs de collecte d'aérosols et de mesure, de façon à toujours disposer de résultats significatifs, malgré la baisse régulière de l'activité de l'air au point d'atteindre aujourd'hui des très bas niveaux. Cet effort continu permet de disposer aujourd'hui d'une précieuse chronique d'observations de 


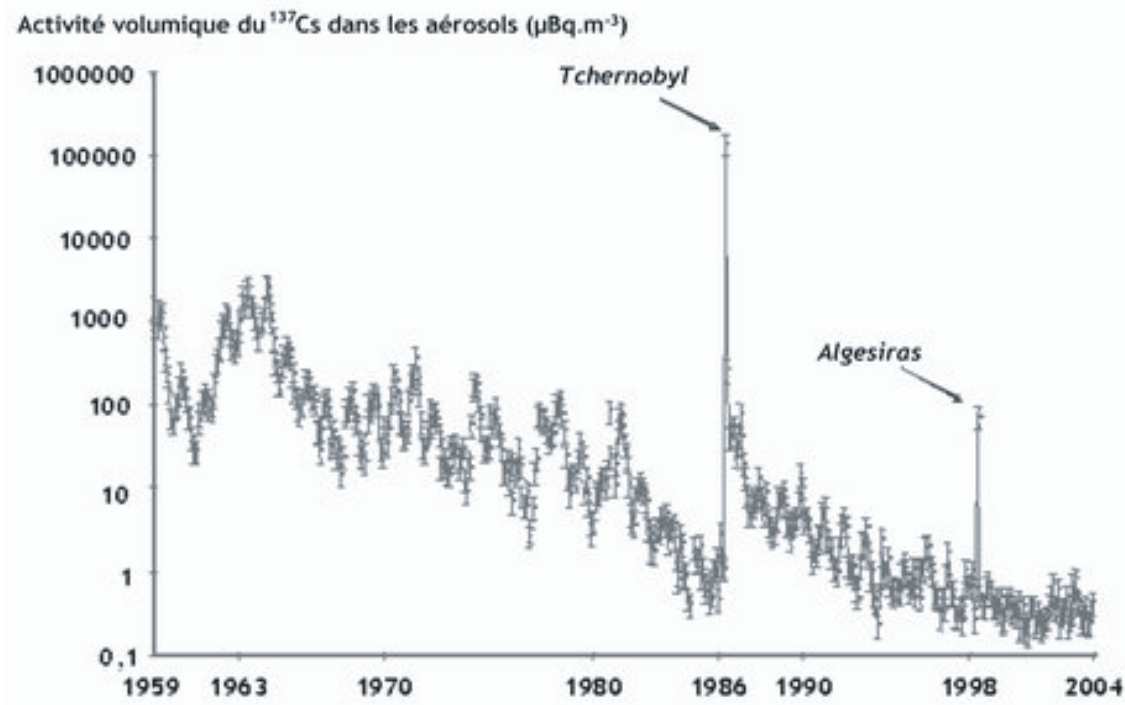

Figure 4-Évolution de l'activité volumique moyenne du césium-137 dans l'air entre 1958 et 2004, observée par les stations OPERA atmosphériques. La période 1958-1980 montre des activités élevées et fluctuantes, en rapport avec les essais nucléaires atmosphériques.

Evolution of the average activities of cesium-137 in the air between 1958 and 2004, observed by OPERA stations. The period 1958-1980 shows high and fluctuating activities in relation to atmospheric nuclear tests.

l'activité du césium-137 dans l'air, illustrée par la figure 4 (Bouisset et al., 2004). Il en est de même pour l'observation de la radioactivité dans les autres milieux ;

- la réalisation de "points zéro » radiologiques, du suivi radioécologique annuel et des bilans décennaux autour des centrales nucléaires. Cette activité régulière menée par l'IRSN autour des centrales nucléaires d'EDF s'inscrit historiquement dans le cadre de conventions d'étude passées avec cette entreprise depuis le début des années 90 et partiellement renouvelées à partir de 2008. Ces études, qui complètent la surveillance réglementaire réalisée par EDF autour de ses sites, permettent de suivre, dans la durée, l'évolution de l'état radiologique autour des sites ; elles donnent lieu à des rapports annuels et à une exploitation à des fins scientifiques ;

- la réalisation d'études environnementales en «zones ateliers », visant à caractériser et comprendre l'origine et les mécanismes de transfert des substances radioactives présentes dans les différents milieux. Ces études sont menées en France ou à l'étranger, soit sur des territoires non directement influencés par les rejets des installations nucléaires actuelles (étude des retombées globales), 
soit au contraire autour de sites nucléaires afin de caractériser l'état radiologique induit par ces activités (plutonium autour de Marcoule, tritium autour de Valduc, carbone-14 autour des centrales nucléaires, etc.) ;

- la réalisation d'un suivi environnemental annuel en Polynésie française, afin d'estimer et de suivre dans la durée les doses reçues par la population.

La plupart des mesures de radioactivité nécessaires à ces différentes études doivent permettre d'accéder à des valeurs significatives à très bas niveau ; à cet effet, ces mesures sont effectuées par le Laboratoire de mesure de la radioactivité de l'environnement (LMRE) de l'IRSN à Orsay, spécialisé dans la réalisation de telles mesures.

Aujourd'hui, l'IRSN bénéficie de cet héritage mixte et enrichissant de l'OPRI et de l'IPSN et l'a intégré dans son organisation interne, mise en place en 2003 à la suite de la création de l'institut. Ainsi :

- le Service d'étude et de surveillance de l'environnement (SESURE) regroupe des équipes venant à la fois de l'OPRI (Le Vésinet) et de l'IPSN (Cadarache, la Seyne-sur-Mer et Tahiti), pour mener conjointement les activités de surveillance de la radioactivité de l'environnement (développement et exploitation des réseaux de prélèvement et de mesure; exploitation et valorisation des résultats) et les activités d'études radioécologiques sur le terrain. Ce rapprochement d'activités a permis de mieux les articuler, de fédérer les compétences, d'optimiser l'utilisation des moyens et de jouer des complémentarités d'approches ;

- le Service de traitement des échantillons et de métrologie de l'environnement (STEME) regroupe un ensemble de laboratoires de traitement radiochimique des échantillons et de mesure d'activité, situés au Vésinet (ex-OPRI) et à Orsay (ex-IPSN) et bénéficiant d'accréditations du COFRAC pour divers types d'analyses. À ce titre, ce service est garant de la qualité des résultats de mesure obtenus dans le cadre de la surveillance de l'environnement ou des études radioécologiques.

L'activité de surveillance proprement dite mobilise en permanence près de 40 salariés de l'IRSN, dont plus de 2/3 sont des techniciens. En complément, les études radioécologiques menées sur le terrain mobilisent plus d'une trentaine de salariés de l'IRSN, dont environ la moitié est constituée d'ingénieurs et de chercheurs.

\section{Les nouveaux défis et les perspectives pour l'IRSN}

Comme on vient de le voir, la surveillance de la radioactivité de l'environnement et les études conduites par l'IRSN sont solidement ancrées dans l'histoire et 
bénéficient d'une grande expérience et d'une bonne maîtrise technique. Pour autant, la question de la surveillance de l'environnement n'est pas dépourvue de défis nouveaux, liés aux évolutions techniques et aux changements de contexte, notamment en termes de transparence :

- d'une part, les chroniques de mesures de la radioactivité en France montrent une tendance générale à la diminution de l'activité de la plupart des radionucléides artificiels dans l'environnement, en relation avec l'évolution des activités nucléaires dont les rejets ont été significativement réduits ;

- d'autre part, des évolutions techniques sont nécessaires pour répondre à des demandes nouvelles des autorités et des acteurs de la société (meilleure capacité de détection), ainsi qu'au vieillissement des équipements en place (perte progressive de leur fiabilité).

Ainsi, le Contrat d'objectifs de l'IRSN 2006-2009, signé par ses cinq ministres de tutelle, prévoit explicitement l'engagement d'un plan de modernisation et d'optimisation de ses réseaux de surveillance, fondé sur une stratégie actualisée et incluant le renforcement de la valorisation des résultats de la surveillance.

Depuis 2006, l'IRSN a engagé des réflexions et des développements techniques répondant à cet engagement, selon deux axes complémentaires :

- une modernisation et un redéploiement de l'ensemble des réseaux permanents de stations de prélèvements et de mesures ayant un rôle de « sentinelle » à la fois autour des sites nucléaires et sur l'ensemble du territoire national ;

- la mise en place d'une surveillance flexible, dans l'espace et le temps, visant à compléter et actualiser la connaissance de l'état radiologique de l'environnement, à expliquer l'origine des radionucléides décelés au voisinage des activités nucléaires actuelles ou passées et, le cas échéant, à investiguer des situations jugées inhabituelles ou anormales afin d'en évaluer l'impact réel. Ce mode de surveillance impose, dans la mesure du possible, de mobiliser les meilleures techniques de prélèvements et d'analyses disponibles.

Les orientations essentielles retenues dans le cadre de ces réflexions et développements sont présentées dans les paragraphes suivants, pour ce qui concerne :

- la modernisation technique des moyens de surveillance de la radioactivité de l'air et l'évolution de l'implantation des balises de prélèvement et de mesure ;

- l'évolution du dispositif de surveillance de l'IRSN autour des sites nucléaires ;

- la surveillance assurée par l'IRSN sur le reste du territoire français ;

- les besoins de développement en métrologie environnementale ;

- l'exploitation et la restitution des résultats de surveillance et l'implication des parties prenantes. 
Chacun pourra constater que la plupart de ces points dépassent le seul cadre d'action de l'IRSN et justifient en conséquence un dialogue soutenu avec les différentes parties prenantes: administrations de l'État, autorités de contrôle, exploitants, collectivités locales, associations, partenaires étrangers, etc.

\subsection{Quelle place pour la surveillance de la radioactivité de l'air?}

L'essentiel de la surveillance de la radioactivité réalisée par l'IRSN est consacré au milieu atmosphérique. On en comprend aisément les raisons historiques, mais l'IRSN a estimé nécessaire d'engager en priorité une réflexion sur le devenir de cette surveillance, pour les raisons suivantes :

- le réseau TELERAY, opérationnel depuis plus de 15 ans, commence à être atteint d'obsolescence, tant pour ce qui concerne les sondes de mesure (sensibilité de mesure faible au regard de celle des sondes modernes, fiabilité progressivement réduite par le vieillissement des équipements) que les moyens de télétransmission et de traitement des résultats de mesure ;

- il en va de même pour le réseau SARA, dont les capacités techniques sont à la limite de ce que l'IRSN estime nécessaire pour caractériser une contamination accidentelle de l'air ;

- le réseau «AS» de prélèvement d'aérosols présente des contraintes d'exploitation lourdes (changement des filtres de prélèvement et envois postaux quotidiens par des opérateurs locaux) et permet une connaissance a posteriori de la contamination de l'air qui mérite une amélioration des limites de détection des radionucléides émetteurs gamma, afin de mieux déceler des événements de faible importance dans l'environnement ;

- l'implantation des stations de prélèvements et de mesures de la radioactivité de l'air, est à ajuster, de manière optimale, en tenant compte des sources d'émission de radioactivité, des lieux où se trouvent des populations « cibles » et de l'exploitation des résultats de mesure dans une démarche d'expertise utilisant des outils de modélisation.

La conduite de cette réflexion est guidée par deux considérations a priori contradictoires :

- d'un côté, dans un contexte de décroissance historique de la radioactivité de l'air (Fig. 4), résultat combiné de la fin des essais nucléaires atmosphériques, de l'absence d'accident nucléaire majeur récent et de l'effort de réduction des rejets atmosphériques de la part des industriels, la connaissance des niveaux d'activité réels de l'air nécessite des techniques de métrologie de plus en plus sophistiquées pour obtenir des mesures à très bas niveau ; de plus, l'exposition directe aux substances radioactives présentes dans l'air constitue actuellement une part marginale des doses reçues par la population, si on excepte le cas spécifique du radon et de ses descendants. Ces constats pourraient inciter à 
réduire l'effort de surveillance de la radioactivité de l'air au moyen de balises fixes ;

- d'un autre côté, l'expérience de l'accident de Tchernobyl et l'étude des scénarios accidentels pouvant affecter les installations nucléaires françaises, sans parler des dispersions accidentelles (ex. incinération) ou malveillantes de sources radioactives diffuses, montrent un enjeu important, en termes de radioprotection, en cas de contamination accidentelle de l'air pouvant entraîner des doses élevées (plusieurs dizaines de millisieverts, voire plus) dans des délais courts (quelques heures) et sur de courtes durées (quelques heures à quelques jours). Dans une telle situation, les moyens de surveillance mis en œuvre doivent permettre :

(1) une détection rapide de la survenue d'une contamination accidentelle de l'air (rôle d'alerte) ;

(2) une caractérisation en temps réel de la contamination de l'air (expertise de crise et actions de protection d'urgence) ;

(3) le recueil d'informations représentatives dans l'espace et dans le temps permettant, après coup, une reconstitution fiable de la dispersion des polluants radioactifs, de l'exposition des personnes au panache radioactif et des retombées radioactives au sol consécutives à cette dispersion atmosphérique.

Ce sont ces dernières considérations, complétées par les recommandations de son conseil scientifique en mars 2006, qui ont conduit l'IRSN à redéfinir sa stratégie de surveillance de la radioactivité de l'air et à mettre en place un vaste programme de modernisation et de redéploiement de son dispositif de surveillance, comprenant principalement :

- le test du comportement de différents types de sondes de mesure du débit de dose ambiant puis le choix d'un modèle de sonde pour remplacer celles du réseau TELERAY; en complément, une étude de dimensionnement de ce réseau, actuellement en cours, pourrait conduire à doubler le nombre de points de mesure sur le territoire (entre 300 et 400). Il faut souligner que dans la plupart des autres pays européens, et dans tous les pays nucléarisés des dispositifs d'alerte de même nature sont déployés depuis de nombreuses années. Ils sont essentiellement sous la responsabilité des autorités ou d'organismes publics, au voisinage des installations et sur l'ensemble des territoires. Certains pays ont une couverture dense, établie par quadrillage (Autriche, Belgique, Finlande). Au premier plan, on trouve le réseau allemand qui est le plus dense d'Europe avec 2150 balises. D'autres ont des réseaux extrêmement ténus ou épars (Portugal, Slovaquie). La densité de la couverture du territoire français est l'une des plus faible, surtout si on la compare à la densité du parc nucléaire (De Cort, 2007). Enfin, quelques pays complètent ce 
type de réseau fixe par des balises mobiles; c'est le cas des États-Unis qui disposent d'un parc de plusieurs dizaines de balises mobiles (Wolbarst et al., 2008). L'ensemble de ces pays ont commencé la rénovation de leurs réseaux d'alerte historiques ;

- le développement d'une nouvelle génération de balises automatisées de prélèvement et de mesure des aérosols de l'air (SARA-II) répondant mieux aux besoins de dépistage et de caractérisation des contaminations atmosphériques accidentelles importantes, et une étude du nombre optimal de balises et de leur implantation territoriale, à l'aide de modèles de dispersion atmosphérique appliqués aux sources potentielles d'émission. Des équipements de même nature sont aussi à l'étude à l'étranger ;

- une redéfinition du rôle du réseau « $\mathrm{AS}$ », accompagnée d'améliorations techniques (notamment par une augmentation des débits de pompage, actuellement d'une dizaine de $\mathrm{m}^{3} / \mathrm{h}$, et par un préleveur automatique réduisant la fréquence d'intervention des opérateurs locaux) ; la réflexion menée à ce sujet va conduire l'IRSN à revoir progressivement l'implantation de certaines des stations et à changer le type de mesure réalisée sur les filtres de prélèvement (arrêt de la plupart des mesures d'activité bêta globale au profit de la spectrométrie gamma). Ces évolutions devraient permettre au réseau « AS » de conserver son rôle actuel de caractérisation d'événements de faible ampleur qui n'entraînent pas des problèmes significatifs de radioprotection à court terme. En situation accidentelle, bien que ce réseau n'ait pas de rôle opérationnel immédiat pour caractériser le panache radioactif en cours de dispersion, sauf en cas de rejet sur plusieurs jours, il devrait permettre de suivre l'évolution de la contamination de l'air au cours du temps et de réaliser des séries pertinentes de prélèvements d'aérosols lors d'une telle situation, pour compléter a posteriori l'expertise des conséquences radiologiques et dosimétriques de l'accident ou de l'incident. Dans cette optique, l'IRSN envisage également de constituer un parc de balises mobiles pouvant être déployées rapidement en cas d'accident.

Les stations OPERA «atmosphériques », dont le débit de pompage est significativement plus élevé (entre 500 et $700 \mathrm{~m}^{3} / \mathrm{h}$ ), conserveront leur rôle actuel « d'observatoire de recherche en environnement »(ORE) destiné à poursuivre la chronique de mesure des contaminants atmosphériques et à fournir un support aux recherches sur les phénomènes de transport atmosphérique et de retombées au sol des poussières radioactives. Leur intégration dans le dispositif de surveillance des aérosols permet de mutualiser et d'améliorer les performances de l'ensemble de ce dispositif. Il faut souligner que les réseaux de surveillance déployés à l'étranger intègrent aussi des équipements présentant des performances similaires. Au-delà du rôle de sentinelle que ces stations peuvent jouer vis-à-vis de la détection d'une 


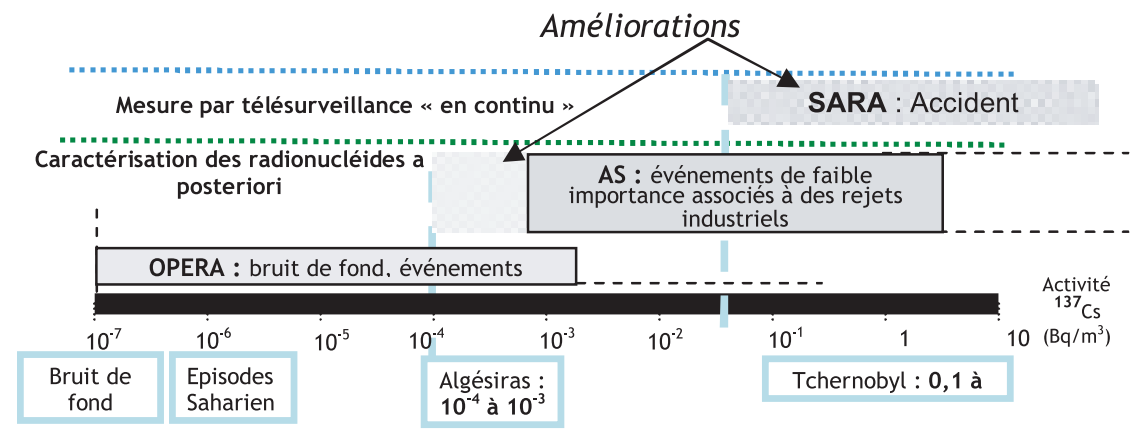

Figure 5 - Gammes des activités volumiques associées aux différents réseaux de surveillance de la radioactivité des aérosols (pour le césium-137).

Range of activity concentrations associated with different networks to monitor the radioactivity of aerosols (for cesium-137).

faible augmentation de la radioactivité, due à des rejets accidentels éloignés de nos frontières, les performances de ces stations OPERA peuvent aussi contribuer au suivi du retour à la situation initiale après un événement radiologique accidentel et à la détection d'évènements naturels.

Le schéma de la figure 5 permet de comparer les performances respectives des différents réseaux de surveillance des aérosols et de montrer leur complémentarité, en termes de gamme de mesure.

\subsection{Quelle implication de l'IRSN dans la surveillance autour des sites nucléaires?}

Actuellement, la surveillance réalisée par l'IRSN concerne, pour une part significative, les sites nucléaires et comprend typiquement :

- la mesure dans l'air du rayonnement gamma ambiant par une sonde TELERAY (mesure permanente ponctuelle) et le déploiement de dosimètres thermoluminescents (mesures intégrées) ;

- le prélèvement de l'air, au niveau du sol, sous les vents dominants de l'installation surveillée (balises AS) ;

- le prélèvement d'eaux de pluies sous les vents dominants par un collecteur ;

- le prélèvement d'eaux souterraines, pour certaines installations ;

- le prélèvement de lait dans une exploitation agricole implantée sous les vents dominants ;

- le prélèvement d'indicateurs biologiques marins côtiers à proximité des installations en bord de mer ; 
- le prélèvement dans le milieu (fluvial ou marin) recevant les rejets liquides de l'installation, assuré par un hydrocollecteur implanté en aval de l'installation, associé à un prélèvement de matières en suspension et complété par un prélèvement d'eau en amont du point de rejet (en cours d'implantation).

Ce plan type est complété, le cas échéant, par des prélèvements de sols, de végétaux et de productions agricoles, réalisés dans le cadre d'études spécifiques.

Une telle surveillance est assurée :

- autour des installations du cycle du combustible (32 sites) : 6 anciens sites miniers, usines de l'amont du cycle du combustible (y compris le site de Malvési), centrales nucléaires et installations de traitement du combustible usé ou de gestion des déchets radioactifs ;

- autour des centres d'études comportant des installations nucléaires $\left(\mathrm{CEA}^{5}\right.$, $\mathrm{CERN}^{6} \ldots$... , y compris avec un statut d'INBS, représentant au total 9 sites ;

- autour des 3 bases navales nucléaires.

Pour une part importante, cette surveillance est redondante de celle réalisée par l'exploitant de l'installation, ce dernier assurant généralement une couverture plus dense à proximité de son site. Par ailleurs, comme déjà indiqué plus haut, les prélèvements d'échantillons mesurés par l'IRSN sont le plus souvent assurés par les services techniques de l'exploitant. Dans ces conditions, la surveillance actuellement assurée par l'IRSN à proximité des sites nucléaires s'apparente à un contrôle croisé des mesures faites par l'exploitant et apporte peu de complémentarité, en termes de connaissance sur l'impact des installations, par rapport aux propres actions des exploitants. C'est pourquoi l'IRSN estime nécessaire de poursuivre une réflexion avec l'ASN ${ }^{7}$ et les exploitants nucléaires, visant à augmenter la complémentarité des dispositifs de surveillance autour des sites nucléaires tout en conservant un minimum de vérification de la surveillance réglementaire réalisée par les exploitants. Les principes directeurs sur lesquels l'IRSN réfléchit actuellement sont les suivants :

- surveillance permanente à proximité des sites : considérant que l'exploitant nucléaire est réglementairement en charge d'assurer une surveillance permanente de la radioactivité en de nombreux points à proximité de ses installations, la surveillance permanente assurée par l'IRSN sur le même territoire ne devrait concerner qu'un nombre plus réduit de points, au titre du contrôle croisé, et être ciblée sur la détection de rejets en situation d'incident ou d'accident (une balise TELERAY, une station AS et des hydrocollecteurs

\footnotetext{
5 Commissariat à l'énergie atomique.

Organisation européenne pour la recherche nucléaire.

Autorité de sûreté nucléaire.
} 
« amont» et «aval»); en contrepartie, l'IRSN devrait pouvoir accéder en permanence aux résultats de la surveillance de l'exploitant, afin de mener à bien les études nécessaires à l'exercice de ses missions, notamment en cas d'événement anormal ;

- campagnes régulières de prélèvements autour des sites nucléaires : l'IRSN devrait réaliser, selon des techniques et des moyens spécifiques n'impliquant pas les exploitants, des campagnes régulières (annuelles ou semestrielles) de prélèvements d'échantillons constituant des indicateurs environnementaux pertinents (végétaux terrestres et aquatiques, productions animales), selon une démarche qui tienne compte de la sensibilité radioécologique des territoires locaux pouvant se trouver sous l'influence des rejets des installations nucléaires. L'objectif de ces campagnes serait de déceler d'éventuelles traces de radionucléides provenant des rejets chroniques des installations, mais aussi de mieux connaître la situation radiologique de «cibles» sensibles au voisinage des sites nucléaires (exploitations de ressources, lieux de vie, etc.). Ces campagnes régulières viendraient compléter les conclusions tirées de la surveillance réglementaire effectuée par les exploitants, ainsi que des suivis radioécologiques annuels réalisés à l'initiative de certains exploitants ;

- capacité de détection et de caractérisation des contaminations atmosphériques d'origine accidentelle : compte tenu du dispositif de surveillance assez complet mis en place par les exploitants à proximité immédiate des sites nucléaires (par exemple entre 8 et 24 balises de mesure du débit de dose ambiant dans un rayon de 5 à 10 kilomètres autour des CNPE), l'IRSN envisage de compléter son dispositif actuel de télésurveillance en le renforçant en priorité dans les sites urbains situés dans un périmètre de quelques dizaines de kilomètres autour des CNPE. Ces territoires qui pourraient être affectées par des dépôts en situation accidentelle, ne bénéficient actuellement de pratiquement aucune surveillance permanente. Ceci pourrait conduire l'IRSN à y installer quelques balises TELERAY ( $c f$. ci-dessus) et certainement une station de type SARA-II, ainsi qu'à y redéployer certaines stations AS ;

- réalisation et mise à jour des états radioécologiques de référence («points zéro ») : l'exploitant devrait continuer à assurer la responsabilité du maintien de ces « points zéro », périodiquement actualisés et aussi complets que possible autour de chaque site nucléaire, comme le fait actuellement EDF lors des bilans environnementaux décennaux. En complément, l'IRSN envisage de réaliser des « constats radiologiques régionaux », sur un «territoire plus étendu que le seul voisinage des installations nucléaires. Ces constats, qui pourraient être actualisés tous les 5 ans environ, auraient pour objectifs, d'une part de caractériser l'influence des installations nucléaires de la région ainsi que la rémanence des retombées atmosphériques anciennes ou à l'éventuelle présence de sources diffuses de radionucléides, d'autre part de disposer d'un référentiel étendu sur 
l'état radiologique des milieux et des denrées alimentaires produites sur ces territoires, utile à la réalisation des expertises en cas de rejet accidentel. La réalisation de tels constats viserait aussi à consolider l'estimation des doses reçues par la population liées aux expositions environnementales ; ces constats devraient également avoir une portée explicative, ce qui signifie que toute « anomalie » décelée à cette occasion devrait donner lieu à des investigations complémentaires destinées à en expliquer l'origine.

En ce qui concerne le cas particulier des nappes phréatiques, la détection précoce des pollutions par des sources pouvant se trouver sur des sites nucléaires, doit rester de la responsabilité première des exploitants nucléaires, comme c'est le cas aujourd'hui. Cette surveillance repose principalement sur des contrôles effectués dans des piézomètres installés dans l'emprise des sites ou à proximité immédiate. Il n'est pas exclu qu'au titre d'un contrôle croisé organisé par les autorités concernées, l'IRSN effectue une analyse des eaux de ces piézomètres, mais cette action devrait rester limitée, sans caractère permanent. Par contre, l'institut propose de développer son action de surveillance sur les utilisations individuelles de l'eau au voisinage des sites nucléaires à l'occasion des constats radiologiques régionaux évoqués précédemment. En ce qui concerne les eaux potables distribuées par le réseau public, dont une part importante vient de nappes phréatiques, il convient également de tenir compte des dispositions réglementaires déjà en place au titre du code de la santé publique, qui impose aux distributeurs d'eau potable de réaliser annuellement un contrôle de la qualité radiologique de leurs eaux ; cette obligation vaut évidemment pour les eaux distribuées sous et hors influence des sites nucléaires.

D’une manière générale, ces principes directeurs développés ci-dessus auront pour effet à terme :

- la réalisation d'actions de surveillance menées par l'IRSN autour des sites nucléaires faisant moins souvent appel au concours technique des exploitants d'INB ou d'INBS, sauf pour ce qui concerne le maintien des dispositifs techniques de surveillance ;

- l'orientation plus marquée du dispositif de surveillance de l'IRSN vers la protection des populations et des éléments les plus sensibles de l'environnement, en modérant la redondance actuelle des mesures à proximité des sources de rejets radioactifs des installations nucléaires (sauf pour la détection des rejets accidentels), compte tenu de la surveillance que l'exploitant est tenu d'assurer par lui-même. 


\subsection{Quelle surveillance sur les territoires éloignés des installations nucléaires?}

Au-delà des territoires accueillant des installations nucléaires et des grandes agglomérations dotées de services de médecine nucléaire, l’IRSN réfléchit à la façon optimale d'assurer une surveillance pertinente sur le reste du territoire, $a$ priori non influencé par des sources importantes de rejets liés aux activités nucléaires. On peut admettre qu'un réseau dense assurant une vigilance permanente n'est pas justifié ici. Néanmoins, deux considérations devraient servir à définir le dispositif de surveillance :

- comme pour les autres parties du territoire, il est nécessaire de disposer de données permettant d'évaluer les doses reçues par la population, en tenant compte notamment de la rémanence des retombées atmosphériques ou des particularités du fond géochimique naturel local; toutefois, ces données devraient être peu fluctuantes au cours du temps, en dehors du cas particulier du radon et de ses descendants ;

- en cas d'accident nucléaire survenant en France ou à l'étranger, ces territoires seraient, comme les autres, susceptibles d'être affectés par un panache radioactif, même de faible activité, entraînant des retombées au sol. L'expérience tirée de l'accident de Tchernobyl montre la grande utilité de disposer d'un minimum de mesures représentatives de la contamination radioactive de l'air, afin de réaliser une reconstitution suffisamment rapide et précise, à l'aide de modélisations, des conséquences radiologiques et dosimétriques de l'accident.

Ces arguments devraient conduire l'IRSN à définir une implantation optimisée de balises TELERAY et de stations de mesure des aérosols (de type SARA-II et AS) dans les territoires dépourvus d'activités nucléaires proches.

En ce qui concerne la connaissance des expositions, outre les informations apportées par la mesure du débit de dose ambiant assurée par le réseau de dosimètres thermoluminescents, qui serait également très utile en situation accidentelle ou post-accidentelle, l'IRSN prévoit de réaliser des campagnes de surveillance de certaines denrées alimentaires, notamment des céréales et du lait en milieu terrestre, des bioindicateurs animaux (poissons, coquillages, etc.) en milieu aquatique et marin, ainsi que des denrées naturelles sensibles (champignons, gibier) dans les régions marquées par des dépôts radioactifs rémanents. L'IRSN est actuellement en contact avec la Direction générale de l'alimentation, l'AFSSA ${ }^{8}$ et la DGCCRF $^{9}$ afin d'intégrer sa démarche de surveillance dans un dispositif plus global d'observatoire de l'alimentation.

8 Agence française de sécurité sanitaire des aliments.

9 Direction générale de la concurrence, de la consommation et de la répression des fraudes. 


\subsection{Quels besoins de développements en métrologie environnementale?}

Les développements de la surveillance de la radioactivité de l'environnement sont fortement liés à ceux des techniques de mesure in situ ou en laboratoire. Les réflexions en cours à l'IRSN, mais aussi celles menées dans le cadre de groupes de travail nationaux ou internationaux (CODIR-PA ${ }^{10}$, OSPAR Commission ${ }^{11}$, etc.), conduisent à dégager les pistes suivantes, non exhaustives, qui s'adressent en premier lieu à l'IRSN et vraisemblablement à l'ensemble des acteurs de la mesure : - les évolutions projetées de la surveillance régulière et des études radioécologiques devraient principalement conduire l'IRSN à réaliser moins d'analyses de strontium- 90 ou d'activités alpha et bêta globales, mais davantage de mesures de carbone-14 (selon des techniques suffisamment précises) et de tritium. Certains radionucléides se prêtent difficilement à la mesure, alors qu'il serait utile de mieux connaître leur devenir dans l'environnement. Il en est ainsi de certains radionucléides émetteurs bêta (nickel-63, chlore-36, etc.), mais aussi, de façon plus courante, du tritium pour lequel l'IRSN prévoit d'appliquer des techniques de mesure avec des limites de détection plus basses, afin de disposer de séries de mesure plus informatives sur ce radionucléide. De plus, l'état des rejets radioactifs dans l'environnement devrait conduire l'IRSN à accroître ses capacités de mesure du krypton-85, du carbone-14 et du tritium dans l'air, ainsi que de l'uranium dans tous les compartiments ;

- dans un contexte où l'environnement radiologique est de plus en plus « propre », les laboratoires de mesure ont pris l'habitude de faire, en routine, des mesures sur des échantillons «non contaminés » ou contaminés à très bas niveau, et sur un nombre limité de radionucléides. Ces laboratoires pourraient avoir des difficultés à interpréter les spectres complexes de radionucléides qui seraient mesurés dans les échantillons contaminés par les rejets accidentels d'un réacteur nucléaire (mélange de radionucléides variés, dont de nombreux à vie courte inhabituellement observés dans l'environnement). De même, la plupart des laboratoires ne sont pas préparés à la mise en place rapide d'une logistique d'accueil et de vérification d'un flux important d'échantillons destinés au contrôle et, en particulier, de s'assurer que l'activité de ces échantillons est bien compatible avec leurs gammes analytiques et la radioprotection du personnel. Face à ce constat, l'IRSN a engagé deux actions :

(1) la réalisation d'exercices d'entraînement de ses laboratoires à l'interprétation de spectres complexes ; à l'avenir, ces exercices pourraient être ouverts à des laboratoires extérieurs volontaires ;

\footnotetext{
${ }^{10}$ Comité directeur pour la gestion de la phase post-accidentelle.

11 OSPAR pour « Oslo-Paris ».
} 
(2) dans le cadre des travaux du CODIR-PA, la rédaction d'un guide de bonnes pratiques des laboratoires de mesure en situation postaccidentelle ;

- les techniques de mesure de routine des radionucléides émetteurs alpha ou bêta donnent des résultats dans des délais longs, susceptibles de retarder les processus d'expertise et les actions de contrôle en situation post-accidentelle. Il est donc souhaitable de poursuivre le développement et la mise en place de techniques d'analyse plus rapides, qui incidemment pourraient s'avérer économiquement favorables dans le contexte de la surveillance de routine.

Ces quelques exemples montrent qu'il existe une large place à l'amélioration des techniques de mesure de la radioactivité dans l'environnement, avec un double intérêt : améliorer la performance des techniques, à la fois pour faire face à une éventuelle situation post-accidentelle et pour compléter les connaissances sur le devenir de certains radionucléides rejetés habituellement dans l'environnement ; réduire les coûts d'analyse en laboratoire grâce à des techniques plus rapides, consommant moins de réactifs chimiques ou de main d'œuvre.

\subsection{Comment mieux répondre aux souhaits et aux besoins d'information des acteurs de la société?}

Malgré les efforts accomplis en matière de surveillance de la radioactivité de l'environnement, de nombreux acteurs locaux et nationaux (y compris au sein des services de l'État) non directement impliqués dans le domaine nucléaire ressentent une insuffisance d'informations et de compréhension à ce sujet, avec un préjugé souvent négatif lié à la perception courante de la façon dont ont été traitées en France les conséquences radiologiques de l'accident de Tchernobyl. Schématiquement, les acteurs de la société posent trois questions :

- comment s'assurer qu' on va surveiller au bon endroit ?

- comment avoir accès à l'ensemble des résultats de mesure dans l'environnement?

- comment interpréter et comprendre la masse de données et d'information délivrée par les experts?

Ces questions s'adressent à tous et, pour sa part, l'IRSN s'efforce d'y apporter une réponse par les actions suivantes :

- mise au point de méthodes d'analyse et recueil de données territoriales prenant en compte la sensibilité environnementale aux substances radioactives ; l'objectif ici est de chercher à concentrer l'effort de surveillance sur les milieux et les produits les plus sensibles, notamment en lien avec le mode de vie et les activités socio-économiques des acteurs locaux. Une telle approche prendrait tout son sens dans un contexte post-accidentel (c'est l'objet 
d'étude du projet PRIME conduit par l'IRSN de 2007 à 2009 dans la basse vallée du Rhône) mais peut également se révéler pertinente pour organiser la surveillance en situation normale ;

- refonte des modes de restitution des résultats de la surveillance réalisée par l'IRSN : depuis 2004, les bulletins mensuels (tableaux de valeurs) diffusés par l'IRSN (et antérieurement par le SCPRI et l'OPRI) ont été remplacés par un bilan annuel de l'état radiologique de l'environnement, à la fois plus synthétique et plus explicatif. Parallèlement, un effort a été fourni pour donner accès, via Internet, à la plupart des résultats de surveillance de l'environnement mais aussi aux résultats d'études menées par l'IRSN sur la radioactivité de l'environnement. Depuis le premier semestre 2009, un nouveau portail sur la radioactivité de l'environnement a été ouvert par l'IRSN dans le but d'améliorer l'ergonomie de l'accès à une information plus synthétique à ce sujet ;

- développement et exploitation par l'IRSN du réseau national de mesures de la radioactivité de l'environnement (RNM), prévu par le Code de la santé publique et mis en place par un comité de pilotage présidé par l'ASN. Ce réseau, mis en service depuis le 15 janvier 2009, permettra le recueil de résultats de mesure de la radioactivité de l'environnement, notamment ceux produits par les laboratoires agréés dans le cadre des programmes de surveillance réglementaire, et organisera, à partir de 2010, leur restitution vers le public via un site internet dédié ;

- ouverture de l'IRSN aux parties prenantes avec notamment la mise en place d'une «Action pilote environnement Loire » (APEL), définie à la suite d'un séminaire $\mathrm{ANCLI}^{12} / \mathrm{IRSN}_{\text {tenu le }} 24$ mars 2004, afin de mieux prendre en compte le besoin pour les acteurs locaux de disposer, à partir des données existantes, d'une information intelligible, répondant aux questions qu'ils se posent sur la qualité de leur environnement. Cette action pilote, menée en association avec les $\mathrm{CLI}^{13}$ de Saint-Laurent-des-Eaux, Dampierre-en-Burly et Chinon, a notamment conduit l'IRSN à réaliser en 2008 un bilan des acteurs, des réseaux de surveillance et des données concernant la radioactivité de l'environnement sur le bassin versant de la Loire. Cette action pilote a contribué à aider les CLI concernées à développer des compétences locales pour comprendre le dispositif de surveillance de l'environnement et ses résultats. Elle a aidé l'IRSN à mieux comprendre les préoccupations des acteurs locaux et a permis d'améliorer en conséquence la présentation des résultats de la surveillance de l'environnement, notamment dans la cadre du RNM.

\footnotetext{
12 Association nationale des commissions locales d'information.

13 Commission locale d'information.
} 
L'expérience montre que l'association des acteurs de la société civile à la réalisation des mesures conforte la compréhension par le public et la crédibilité des résultats de l'IRSN en tant qu'expert institutionnel. Ainsi, dans le cadre des réflexions en cours sur l'évolution de la surveillance exercée par l'IRSN, l'IRSN va étudier la possibilité d'impliquer des parties prenantes locales dans la réalisation des campagnes annuelles de prélèvements que l'IRSN pourrait mettre en place autour des sites nucléaires, tout en veillant à maintenir la qualité technique des opérations de prélèvement (IRSN, 2008).

On peut donc considérer que ces actions contribuent à une évolution favorable mais les retombées positives, en termes de perception et de compréhension par les différents publics, ne peuvent être espérées que sur le moyen et le long terme, à la suite d'un effort durable.

\section{Conclusion}

Partant d'une histoire et d'une expérience solides, l'IRSN est résolument engagé dans une démarche visant à faire évoluer, à divers titres, son dispositif de surveillance de la radioactivité de l'environnement; cette évolution devrait reposer fondamentalement sur deux approches complémentaires :

- un ensemble de réseaux permanents de stations de prélèvements et de mesures ayant un rôle de «sentinelle» à la fois autour des sites nucléaires et sur l'ensemble de territoire national ; ces réseaux, principalement centrés sur la surveillance des milieux atmosphérique et aquatique, font l'objet d'un important programme de modernisation et de redéploiement qui va se poursuivre sur plusieurs années, en cherchant la complémentarité avec les autres dispositifs de surveillance, principalement ceux des exploitants nucléaires, et une répartition optimisée sur le territoire permettant d'acquérir rapidement un nombre suffisant de données exploitables en situation accidentelle. Cette surveillance régulière par des réseaux fixes devrait être complétée par des « audits environnementaux » sous forme de campagnes annuelles autour des sites nucléaires ;

- une surveillance flexible, dans l'espace et le temps, visant à compléter et actualiser la connaissance de l'état radiologique de l'environnement, à identifier et à expliquer la présence éventuelle de radionucléides à des faibles niveaux, liés aux activités nucléaires actuelles ou passées et, le cas échéant, à investiguer des situations jugées inhabituelles ou anormales afin d'en connaître l'origine et l'impact réel. Ce mode de surveillance doit, dans la mesure du possible, faire appel à des techniques de mesure permettant de quantifier les activités des radionucléides d'intérêt, afin de connaître les tendances temporelles et spatiales et de disposer d'une base de connaissances complémentaire pour conforter l'estimation des doses dues aux expositions environnementales. Cette approche 
«flexible » de la surveillance doit également intégrer le cas des situations postaccidentelles, qui nécessite une préparation spécifique des acteurs et des moyens techniques qui seraient mobilisés dans de telles circonstances.

Les évolutions issues de la réflexion en cours au sein de l'IRSN permettront de concrétiser le rapprochement des compétences et des modes d'action hérités de l'OPRI et de l'IPSN au profit d'une démarche d'ensemble plus complète. Mais ces évolutions ne pourront pas être accomplies par l'IRSN seul et nécessiteront de poursuivre un dialogue avec les prescripteurs et autres acteurs de la surveillance (ASN, services de l'État, exploitants) ainsi que les parties prenantes intéressées par les résultats de la surveillance (agences sanitaires, CLI, collectivités locales, etc.). Enfin, l'IRSN tient compte de la dimension économique de ces évolutions, en recherchant un emploi optimal des moyens qu'il peut consacrer à la surveillance et la complémentarité avec les dispositifs retenus par les autres acteurs concernés.

\section{RÉFÉRENCES}

Bouisset P., Barker E., Masson O., Gurriaran R., Gagnat X., Mekhlouche D., Aubry S., Hadjaj M., Saey L. (2004) Concentration de ${ }^{137} \mathrm{Cs}$ et de ${ }^{7}$ Be dans les aérosols en France métropolitaine et à Tahiti de 1959 à 2002, Radioprotection 39, 367-381.

De Cort M. (2007) Monitoring radioactivity in nuclear and radiological emergency in a European perspective: aims and means, Int. J. Emerg. Manag. 4, 319-388.

IRSN (2008) Rapport pour le Haut comité pour la transparence et l'information sur la sécurité nucléaire, Etat de la surveillance environnementale et bilan du marquage des nappes phréatiques et des cours d'eau autour des sites nucléaires et des entreposages anciens de déchets radioactifs, Rapport IRSN/FRM-285 ind. 2.

Wolbarst A.B., Griggs J., Lee H.N., Ren T., Hudson T., White J.D., Zhu C. (2008) Comparison of environmental radiation monitoring programs in China and the United States, Health Phys. 94, $501-511$. 